\title{
Emotion-Word Processing Difficulties in Abstinent Alcoholics With and Without Lifetime Externalizing Disorders
}

\author{
Michael J. Endres and George Fein
}

\begin{abstract}
Background: Evidence suggests that abstinent alcoholics have difficulties processing a variety of emotion-laden stimuli, and some of these difficulties may not fully resolve with long-term abstinence. The current study examined whether emotion-word processing difficulties were present in long-term abstinent alcoholics (LTAA; 18+ months of sobriety) with and without a previously diagnosed externalizing (EXT; antisocial personality disorder and/or attention-deficit/hyperactivity disorder diagnosis) disorder.

Methods: Subjects $(N=121)$ completed an affective go/no-go (AGNG) task with positive, negative, and neutral emotion-word stimuli, and a lexical decision-making (LDM) task with nonemotion word and nonword stimuli. Nonsubstance abusing controls (NSAC; $n=38,50.0 \%$ women, mean age $=48 \pm 7.8)$, LTAA with EXT $(n=32,41 \%$ women, mean age $=47.1 \pm 6.6)$, and LTAA without EXT $(n=51,47 \%$ women, mean age $=49.7 \pm 6.5)$ were compared between signal discriminability $\left(d^{\prime}\right)$ and mean response times (RT) for correct responses (mcRT).

Results: In the LDM task, LTAA had lower $\left(d^{\prime}\right)$ values and slower mcRT than NSAC. In the AGNG task, LTAA and NSAC did not differ in AGNG task mcRT. LTAA had lower $\left(d^{\prime}\right)$ values than NSAC, and this effect was partially associated with group differences in LDM task $\left(d^{\prime}\right)$ values. In LTAA, lower AGNG $\left(d^{\prime}\right)$ values also were associated with an earlier age of first drink, greater lifetime alcohol use, and a history of EXT disorder.

Conclusions: Our findings suggest that detecting the emotional content of words is impaired in LTAA, and this impairment is over and above LTAA's more general lexical processing difficulties. Results also suggest that specific emotion processing impairments in LTAA may be exacerbated by greater lifetime alcohol use burden and other comorbid EXT diagnoses.
\end{abstract}

Key Words: Alcoholism, Long-Term Abstinence, Externalizing Disorders, Emotion Processing, Discriminability.

A BSTINENT ALCOHOLICS ARE known to be less accurate and/or slower at processing a variety of emotion-laden stimuli, including facial expressions (Fein et al., 2010; Foisy et al., 2007a,b; Frigerio et al., 2002; Kornreich et al., 2001; Marinkovic et al., 2009; Maurage et al., 2009, 2008; Oscar-Berman et al., 1990; Philippot et al., 1999; Uekermann et al., 2005), body postures (Maurage et al., 2009), speech tones (Maurage et al., 2009; Monnot et al., 2001; Uekermann et al., 2005), pictures (Gilman and Homer, 2008), and words (Clark et al., 2007; Stormark et al., 2000). Difficulties decoding emotional facial expressions are well documented in alcoholics with weeks, months, and even years of sobriety. Although evidence has indicated that some difficulties processing emotional faces do not fully resolve with long-term abstinence, it is unclear whether multiyear

From the Neurobehavioral Research, Inc. (MJE, GF), Honolulu, Hawaii.

Received for publication April 18, 2012; accepted September 14, 2012. Reprint requests: Michael J. Endres, Neurobehavioral Research, Inc., 1585 Kapiolani Blvd. Ste. 1030, Honolulu, HI 96814; Tel.: 812-272-4716; Fax: 808-442-1156; E-mail: mendres@nbresearch.com

Copyright $(C) 2012$ by the Research Society on Alcoholism.

DOI: 10.1111/acer.12026 abstinent alcoholics have difficulties processing other types of emotion-laden stimuli. The current research investigated whether emotion-word processing difficulties are present in long-term abstinent alcoholics (LTAA, 18+ months).

Alcoholics who can quit drinking, and remain sober, show significant improvement in some, but not all, domains of neuropsychological functioning. Impairments in higherorder cognition, such as reduced short-term memory and working memory capacity, often normalize with extended abstinence (Bartsch et al., 2007; Fein et al., 2006; Mann et al., 1999; Nixon and Glenn, 1995; Rosenbloom et al., 2004; Sullivan and Pfefferbaum, 2005). In contrast, impairments in emotion perception, such as emotional facial expression decoding deficits, have been consistently found in alcoholics with extended abstinence.

When compared to nonalcoholics, recently detoxified alcoholics (RDA; e.g., 2 to 4 weeks), short-term abstinent alcoholics (STAA) (e.g., 5 to 15 weeks), and LTAA are typically less accurate and/or slower at detecting the valence and intensity of positive (e.g., happiness and surprise) and negative (e.g., fear, anger, disgust, and contempt) facial expressions (Fein et al., 2010; Foisy et al., 2007a,b; Kornreich et al., 2001; Marinkovic et al., 2009; Philippot et al., 1999; Townshend and Duka, 2003). However, methodological 
differences between studies have led to some inconsistencies with regard to the exact characterization of emotional facial expression decoding deficits in abstinent alcoholics. Using a test retest design, Foisy and colleagues (2007a) showed RDA were less accurate than controls at recognizing and estimating the intensity of positive and negative faces, and these differences were still apparent after 2 months of abstinence. Kornreich and colleagues (2001) also showed that RDA, STAA (2 to 6 months), and LTAA (1 to 9 years) had comparable difficulties recognizing negative expressions (e.g., anger, sadness, and disgust), but found no evidence that STAA and LTAA overestimated the intensity of positive and negative expression. Fein and colleagues (2010) showed that LTAA (18+ months) were slower than controls at recognizing positive (i.e., happy), negative (i.e., sad), and neutral facial expressions, but no differences in accuracy were found. Both longitudinal and crosssectional studies have provided evidence to suggest that some difficulties processing emotional faces are present in LTAA, and these impairments may not fully resolve with extended abstinence.

It is unclear whether alcoholics with multiyear abstinence have difficulty processing emotion-laden stimuli other than emotional faces. Maurage and colleagues (2009) found RDA were less accurate than controls at estimating the intensity of emotional faces, postures, and voices, but not written scenarios. In contrast, studies using emotional Stroop-like tasks have shown RDA are typically slower than controls when ignoring positive, negative, and neutral emotion words (Bauer and Cox, 1998; Stormark et al., 2000). Other studies have found that, when compared to controls, STAA (4+ weeks) attribute higher positive valences to positive words and perceive emotional content in neutral words (Clark et al., 2007; for similar findings, also see Steinmetz and Federspeil, 2012). Although this work has provided evidence that emotion-word processing difficulties are present in alcoholics who have just begun the recovery process, previous published reports have not established whether emotion-word processing difficulties are present in alcoholics with multiyear abstinence. The present research investigated emotion-word processing in nonsubstance abusing controls (NSAC) and LTAA using an affective go/no-go (AGNG) task, which assessed the ability to correctly activate and inhibit behavioral responses to positive, negative, and neutral emotion words. General lexical processing abilities also were assessed using a lexical decision-making (LDM) task, which served as a control task and tapped the ability to correctly classify words and nonwords. Based on the evidence from studies using emotional face stimuli, we hypothesized that emotionword processing abilities would be impaired in LTAA and that this impairment would be present even after controlling for individual differences in general lexical processing ability.

It also is unclear whether alcoholics with a history of cooccurring psychiatric disorders experience less recovery of emotion processing abilities than those without such conditions. Studies have shown that impaired judgment of negative-valenced stimuli (e.g., fearful and sad faces) in abstinent alcoholics can be partially explained by the co-occurrence of internalizing (i.e., mood and anxiety) disorders (Gotlib et al., 2004; Townshend and Duka, 2003). Other research has shown impaired judgment of negative-valenced stimuli (e.g., angry and sad faces) in individuals with externalizing (EXT) (i.e., attention-deficit/hyperactivity) disorders (Pelc et al., 2006). Studies also have suggested that emotion processing difficulties in abstinent alcoholics are part of a much broader pattern of disinhibited executive control processes (Marinkovic et al., 2009), which is known be a common psychological characteristic among those with EXT spectrum disorders (Bogg and Finn, 2010; Endres et al., 2011; Finn, 2002). The present research examined whether emotion-word processing difficulties in LTAA were present when controlling for psychiatric comorbidities. We hypothesized that LTAA with a history of internalizing or EXT disorder would show the greatest impairment in emotion-word processing abilities.

\section{MATERIALS AND METHODS}

\section{Participants}

Study participants were recruited using various methods, including participant referrals, Craig's list advertisements, and postings at Alcohol and Narcotics Anonymous meetings, transitional living centers, treatment centers, community centers, and university campuses. A total of 121 subjects (46.3\% women) ranging from 35 to 61 years of age (mean age $=48.5 \pm 7$ ) completed the current study. Of these, 38 subjects were NSAC $(50.0 \%$ women; mean age $=48 \pm 7.8)$, and the remaining 83 subjects were LTAA $(44.6 \%$ women; mean age $=48.7 \pm 6.6$ ).

The NSAC group consisted of participants who (i) did not meet DSM-IV (American Psychiatric Association, 2000) criteria for any substance abuse/dependence disorder (except for nicotine and caffeine abuse/dependence) as ascertained by the computerized Diagnostic Interview Schedule (Robins et al., 1998), (ii) had a lifetime drinking average of $<30$ drinks per month, and (iii) never consumed over 60 drinks in a month. LTAA met DSM-IV criteria for a history of alcohol dependence and had a minimum of 18 months sobriety. Participant's length of abstinence was self-reported.

Exclusion criteria for all groups were the following: (i) significant history of head trauma or cranial surgery or neurological disease, (ii) history of diabetes, stroke, or hypertension that required medical intervention, (iii) laboratory evidence of hepatic disease, (iv) clinical evidence Wernicke-Korsakoff syndrome, (v) currently taking prescribed psychotropic, stimulant, or pain medications, and (vi) lifetime or current diagnosis of schizophrenia or schizophreniform disorder.

\section{LDM Task}

Processing of nonemotion words was assessed with an LDM task. Subjects were presented with a series of words (e.g., full, light, plural) and nonwords (e.g., sles, phypa, taysef) in a pseudorandomized order. Stimuli were displayed sequentially in the center of a computer monitor for $1,000 \mathrm{~ms}$ followed by a 1- to 100 -ms delay. The subject's task was to as quickly and as accurately as possible press a response key for words (e.g., button A) and another response key for nonwords (e.g., button B). A response was recorded only if it occurred within the 1,000-ms stimulus presentation window. Subjects were not given corrective feedback. The LDM task consisted of 215 trials with $20 \%$ reflecting nonwords. 


\section{AGNG Task}

Processing of emotion words was assessed with the AGNG task (Murphy et al., 1999). Part of the Cambridge Automated Neuropsychological Test Battery (Cambridge Cognition Ltd., Cambridge, MA), the AGNG task has been shown to be a reliable assessment of emotion-word processing abilities in a variety of nonclinical (e.g., Robbins et al., 1994) and clinical populations (e.g., Murphy et al., 1999).

Subjects were presented with 2 sets of emotion words: one representing target stimuli, and the other representing distractor stimuli. Target and distractor stimuli had either a positive (e.g., joy, luck, praise), negative (e.g., sad, loss, shame), or neutral (e.g., opt, send, pause) emotional valence. Emotion-word stimuli were not alcohol related. Stimuli were presented one-at-a-time in the center of a computer screen for $300 \mathrm{~ms}$ followed by a $900-\mathrm{ms}$ blank screen. The subject's task was to press a single response key when presented with a target word and inhibit that same response when presented with a distractor word. Subjects were instructed to respond as quickly and as accurately as possible and were not given corrective feedback.

Participants completed 3 different AGNG conditions, hereafter referred to a positive valence blocks (PVB), negative valence blocks (NVB), and mixed valence blocks (MVB). PVB consisted of 2 blocks of trials: one with positive targets and neutral distractors and the other with neutral targets and positive distractors. NVB also consisted of 2 blocks of trials: one with negative targets and neutral distractors and the other with neutral targets and negative distractors. MVB also consisted of 2 blocks of trials: one with positive targets and negative distractors and the other with negative targets and positive distractors. The task consisted of 20 blocks: 6-PVB, 6-NVB, and 8-MVB. Blocks were presented in a pseudorandomized order that was fixed across subjects. Subjects completed 18 trials (9-target trials and 9-distractor trials) per block, such that the task consisted of 360 total trials. Subjects were told whether positive, negative, or neutral affective words would represent target and distractor stimuli prior to each block.

\section{Measures of LDM and AGNG Task Performance}

Accuracy and response times (RT) were obtained from the LDM and AGNG tasks. Mean RT was computed for correct responses only. Task accuracy was assessed using a signal detection theory (Greene and Swets, 1966; MacMillan and Creelman, 1990a,b) measure of perceptual sensitivity (discriminability $\left.\left[d^{\prime}\right]\right)$. The $\left(d^{\prime}\right)$ measure has been shown to be a more psychometrically sensitive and psychologically meaningful measure of decision-making accuracy than correct and incorrect responses alone (Greene and Swets, 1966). Consistent with the univariate Gaussian signal detection theory model, correct responses to target stimuli (hits [HT]) and incorrect responses to distractor stimuli (false alarms [FA]) were converted into proportions and then standardized using an inverse normal probability density function $(\varphi)$ transformation with mean 0 and standard deviation 1. These quantities, denoted $\varphi \mathrm{HT}$ and $\varphi \mathrm{FA}$, respectively, were used to calculate $d^{\prime}$

$$
d^{\prime}=\varphi \mathrm{HT}-\varphi \mathrm{FA} .
$$

Discriminability operationalized the ability to generate accurate internal representations of LDM and AGNG task stimuli. In the LDM task, higher $\left(d^{\prime}\right)$ values reflect an increased perceptual sensitivity to the lexical information conveyed by words and nonwords as indicated by high HT and low FA. In the AGNG task, higher $\left(d^{\prime}\right)$ values reflect an increased perceptual sensitivity to the emotional content of positive, negative, and neutral valenced words as indicated by high HT and low FA.

\section{Procedure}

Participants completed a total of 4 testing sessions, with each session lasting between 1.5 and 4 hours. All participants were informed of the aims and procedures of the current study and signed an informed consent. NSAC participants were asked to abstain from alcohol for at least 24 hours prior to testing. Breathalyzer (Intoximeters, Inc., St. Louis, MO) and Oral Fluid Drug Screen Device (Innovacon, Inc., San Diego, CA) tests were administered to each participant before each session. An alcohol concentration of 0.000 and negative drug screen were required for all participants for all testing sessions. Participants were compensated for their time, reimbursed for travel expenses, and given a bonus for completing the entire study.

\section{Data Analyses}

Multivariate analysis of variance with planned comparisons using SPSS version 18 (SPSS Inc., Chicago, IL) was the primary data analytic approach used.

\section{RESULTS}

\section{Sample Characteristics}

Sample characteristics are reported in Table 1. NSAC and LTAA had comparable gender and age distributions. LTAA had fewer years of education, more dense family drinking

Table 1. Sample $(N=121)$ Demographics, Lifetime DSM-IV Diagnoses, and Lifetime Drinking Variables for NSAC and LTAA

\begin{tabular}{|c|c|c|c|}
\hline Measures & $\begin{array}{c}\text { NSAC } \\
(n=38) \\
\% \text { or } M(\mathrm{SD})\end{array}$ & $\begin{array}{c}\text { LTAA } \\
(n=83) \\
\% \text { or } M(\mathrm{SD})\end{array}$ & $\begin{array}{l}\text { NSAC vs. LTAA } \\
\text { odds or effect }\end{array}$ \\
\hline \multicolumn{4}{|l|}{ Demographics } \\
\hline Gender (\% women) & $50 \%$ & $45 \%$ & 0.31 (ns) \\
\hline Age (years) & $48.16(7.59)$ & $48.63(6.65)$ & $<0.01$ (ns) \\
\hline Education (years) & $16.26(3.02)$ & $13.44(2.25)$ & $19.41 * * *$ \\
\hline \multicolumn{4}{|c|}{ Lifetime DSM-IV diagnoses } \\
\hline $\begin{array}{l}\text { Substance use } \\
\text { disorder }^{\mathrm{a}}\end{array}$ & $0 \%$ & $47 \%$ & NA \\
\hline $\begin{array}{l}\text { Externalizing } \\
\text { disorder }\end{array}$ & $0 \%$ & $39 \%$ & $29.12 * * *$ \\
\hline $\begin{array}{l}\text { Internalizing } \\
\text { disorder }\end{array}$ & $21 \%$ & $61 \%$ & $17.88 * * *$ \\
\hline \multicolumn{4}{|c|}{ Lifetime drinking variables } \\
\hline $\begin{array}{l}\text { Family history of } \\
\text { alcoholism (\% first- } \\
\text { degree relatives) }\end{array}$ & $17 \%(21 \%)$ & $34 \%(29 \%)$ & $7.27 * *$ \\
\hline $\begin{array}{l}\text { Age of first drink } \\
\text { (years) }\end{array}$ & $20.56(8.42)$ & $14.38(4.17)$ & $20.37 * * *$ \\
\hline $\begin{array}{l}\text { Lifetime alcohol use } \\
\text { (standard drinks } \times \\
1,000)^{\mathrm{a}}\end{array}$ & $2.07(0.53)$ & $60.69(58.27)$ & NA \\
\hline $\begin{array}{l}\text { Average alcohol } \\
\text { dose (standard } \\
\text { drinks/month) }\end{array}$ & $9.22(9.60)$ & $204.42(155.42)$ & NA \\
\hline $\begin{array}{l}\text { Duration of } \\
\text { abstinence } \\
\text { (months) }^{\mathrm{a}}\end{array}$ & NA & $91.63(84.26)$ & NA \\
\hline
\end{tabular}

NSAC, nonsubstance abusing controls; LTAA, long-term abstinent alcoholics.

${ }^{\text {a }}$ Statistical comparisons between groups are not valid because the inclusion criterion was associated with this variable. $p$-Value not significant at $p<0.05$ (ns), $* p<0.05, * * p<0.01, * * * p<0.001$. 
histories, and an earlier age of first drink than NSAC. Internalizing and EXT disorders were more prevalent in LTAA than NSAC.

\section{Preliminary Analyses}

Correlations between sample characteristics and AGNG and LDM mean $\left(d^{\prime}\right)$ and correct RT (mcRT) were examined separately for NSAC and LTAA. For NSAC, demographic and lifetime drinking variables were not correlated with AGNG and LDM measures. For LTAA, fewer years of education was associated with lower $\left(d^{\prime}\right)$ values in the AGNG $(r=0.37, p \leq 0.001)$ and $\operatorname{LDM}(r=0.35, p \leq 0.001)$, and earlier age of first drink $(r=0.28, p \leq 0.05)$ and greater lifetime alcohol use $(r=-0.26, p \leq 0.001)$ were associated with lower AGNG $\left(d^{\prime}\right)$ values. These associations were not present in NSAC. Additionally, abstinence duration was not correlated with AGNG and LDM measures in LTAA.

Correlations between AGNG and LDM measures revealed significant effects for $\left(d^{\prime}\right)$ values and mcRT. Higher AGNG task $\left(d^{\prime}\right)$ values were associated with higher LDM task $\left(d^{\prime}\right)$ values in both NSAC $(r=0.33, p \leq 0.05)$ and LTAA $(r=0.46, p \leq 0.001)$. Likewise, higher AGNG task mcRT values were associated with higher LDM task mcRT in both NSAC $(r=0.40, p \leq 0.05)$ and LTAA $(r=0.44$, $p \leq 0.001)$. These effects were controlled for by regressing LDM task $\left(d^{\prime}\right)$ and mcRT values on AGNG task $\left(d^{\prime}\right)$ and mcRT values separately for PVB, NVB, and MVB. The unstandardized residuals were then added to the grand means for PVB, NVB, and MVB to yield adjusted $\left(d^{\prime}\right)$ and mcRT values. Means and standard deviations for AGNG and LDM $\left(d^{\prime}\right)$ values are shown in Fig. 1. Means and standard deviations for AGNG and LDM mcRT are

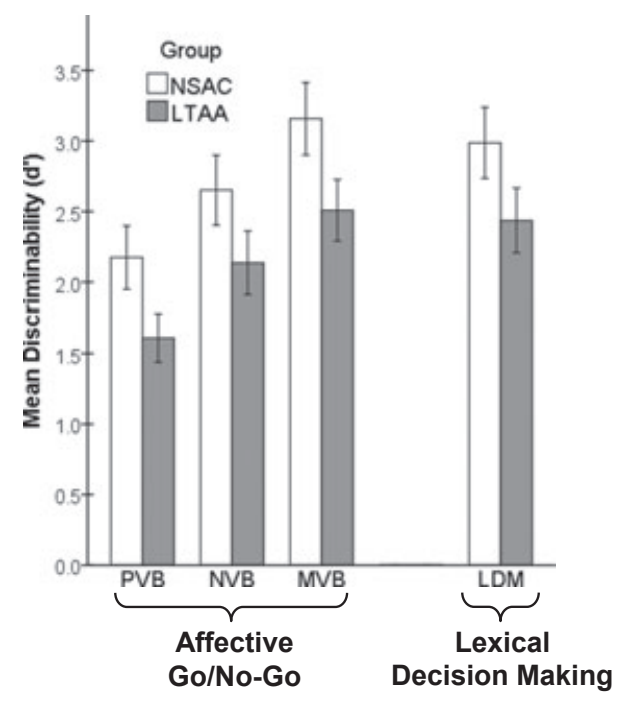

Fig. 1. Bar graphs with $95 \%$ confidence intervals for mean discriminability $\left(d^{\prime}\right)$ values by group membership, lexical decision-making (LDM) task, and affective go/no-go task condition. NSAC, nonsubstance abusing controls; LTAA, long-term abstinent alcoholics; PVB, positive valence blocks; NVB, negative valence blocks; MVB, mixed valence blocks. reported in Table 2. Adjusted means and standard deviations for PVB, NVB, and MVB $\left(d^{\prime}\right)$ values are shown in Figs 2 and 3.

\section{Are Difficulties with Emotion-Word Processing Present in LTAA?}

LTAA had less accurate discrimination of positive, negative, and neutral words than NSAC, but groups did not differ in how fast they discriminated emotion words. In Fig. 1, LTAA had lower unadjusted $\left(d^{\prime}\right)$ values than NSAC in PVB, $F(1, \quad 120)=14.65, \quad p \leq 0.001, \quad \eta^{2}=0.11, \quad \mathrm{NVB}, \quad F(1$, $120)=7.06, \quad p \leq 0.01, \quad \eta^{2}=0.06, \quad$ and MVB, $F(1$, $120)=12.53, p \leq 0.001, \eta^{2}=0.10$. In Table 2, LTAA and NSAC did not differ in unadjusted $\operatorname{mcRT}(p \geq 0.145$, $\left.\eta^{2} \leq 0.02\right)$.

\section{Are Difficulties with LDM Present in LTAA?}

LTAA had less accurate and slower discrimination of words from nonwords in comparison with NSAC. In Fig. 1, LTAA had lower LDM task $\left(d^{\prime}\right)$ values than NSAC, $F(1,120)=8.36, p \leq 0.01, \eta^{2}=0.07$. In Table 2, LTAA also had slower LDM task mcRT than NSAC, $F$ $(1,120)=12.88, p \leq 0.001, \eta^{2}=0.10$.

\section{Are Difficulties with Emotion-Word Processing in LTAA} Entirely Due to More General Difficulties in LDM?

LTAA had less accurate discrimination of positive, negative, and neutral emotion words than NSAC, and this effect was over and above group differences in discrimination of words from nonwords. Specifically, group differences in LDM task $\left(d^{\prime}\right)$ values could account for about half of group differences in AGNG task $\left(d^{\prime}\right)$ values. In Fig. 2, LTAA had lower adjusted $\left(d^{\prime}\right)$ values than NSAC in PVB, F $(1,120)=$

Table 2. LDM and AGNG Task Mean Reaction Time on Correct Trials for NSAC and LTAA

\begin{tabular}{llll}
\hline & $\begin{array}{c}\text { NSAC } \\
(n=38) \\
M(S D)\end{array}$ & $\begin{array}{c}\text { LTAA } \\
(n=83) \\
M(S D)\end{array}$ & $\begin{array}{c}\text { NSAC vs. } \\
\text { LTAA } \\
\text { Effect }\end{array}$ \\
\hline Measures & $569(80)$ & $579(91)$ & $0.32(\mathrm{~ns})$ \\
$\begin{array}{l}\text { AGNG } \\
\text { PVB positive word } \\
\text { vs. neutral word }\end{array}$ & $570(75)$ & $586(91)$ & $0.75(\mathrm{~ns})$ \\
$\begin{array}{l}\text { NVB negative word } \\
\text { vs. neutral word }\end{array}$ & $529(60)$ & $550(80)$ & $1.78(\mathrm{~ns})$ \\
$\begin{array}{l}\text { MVB positive word } \\
\text { vs. negative word }\end{array}$ & & & \\
$\begin{array}{l}\text { LDM } \\
\text { Word vs. nonword }\end{array}$ & $470(68)$ & $520(73)$ & $4.49^{*}$ \\
\hline
\end{tabular}

NSAC, nonsubstance abusing controls; LTAA, long-term abstinent alcoholics; PVB, positive valence blocks; NVB, negative valence blocks; MVB, mixed valence blocks; LDM, lexical decision making; AGNG, affective go/ no-go.

Reaction time is in milliseconds. $p$-Value not significant at $p<0.05$ (ns), $* p<0.05$. 


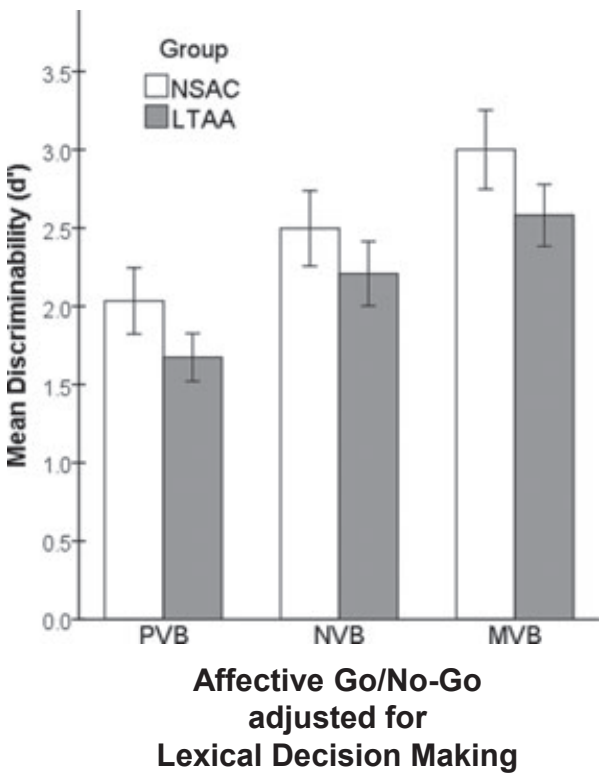

Fig. 2. Bar graphs with $95 \%$ confidence intervals for mean discriminability $\left(d^{\prime}\right)$ values by group membership and affective go/no-go task (AGNG) condition. Participant $\left(d^{\prime}\right)$ values for each AGNG task condition were adjusted to account for lexical decision-making task $\left(d^{\prime}\right)$ values. NSAC nonsubstance abusing controls; LTAA, long-term abstinent alcoholics; PVB, positive valence blocks; NVB, negative valence blocks; MVB, mixed valence blocks.

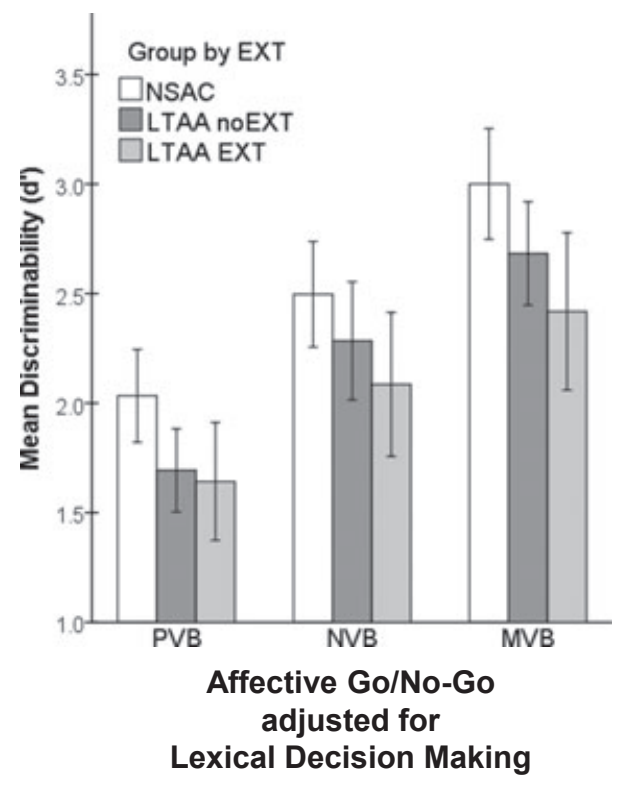

Fig. 3. Bar graphs with $95 \%$ confidence intervals for mean discriminability $\left(d^{\prime}\right)$ values by group membership, externalizing (EXT) diagnosis history, and affective go/no-go task (AGNG) condition. Participant $\left(d^{\prime}\right)$ values for each AGNG task condition were adjusted to account for lexical decision-making task $\left(d^{\prime}\right)$ values. NSAC, nonsubstance abusing controls; LTAA noEXT, long-term abstinent alcoholics without EXT diagnosis; LTAA EXT, long-term abstinent alcoholics with EXT diagnosis.

$7.24, p \leq 0.01, \eta^{2}=0.06$, and MVB, $F(1,120)=6.12$, $p \leq 0.05, \eta^{2}=0.05$, but not NVB, $F(1,120)=2.81$, $p>0.05, \eta^{2}=0.02$.
Are Lifetime Internalizing and EXT Diagnoses Associated with Greater Difficulty Processing Emotion Words in LTAA?

Multivariate tests indicated that there were no omnibus effects of lifetime internalizing disorder (positive or negative), $F(2,117)=0.70, p>0.05, \eta^{2}=0.02$, or group by lifetime internalizing disorder interaction, $F(2,117)=0.71, p>0.05$, $\eta^{2}=0.02$, on LDM and AGNG measures. In contrast, significant omnibus effects of lifetime EXT disorder (positive vs. negative), $F(2,117)=7.24, p \leq 0.001, \eta^{2}=0.14$, on LDM and AGNG measures were found, and these effects were independent of significant group (NSAC vs. LTAA), $F$ $(2,117)=3.41, p \leq 0.05, \eta^{2}=0.08$, effects on LDM and AGNG measures. Test of the 2-way interaction between group and EXT history was invalid because $0 \%$ of subjects in NSAC had a previously diagnosed EXT disorder (see Table 1).

In Table 3, sample characteristics are shown for LTAA split into subgroups with and without a lifetime EXT disorder diagnoses. These subgroups were comparable in gender composition, years of education, history of internalizing disorders, and lifetime drinking variables. LTAA with EXT were older and had a greater lifetime prevalence of other substance use disorders than their counterparts without EXT.

Difficulties discriminating positive, negative, and neutral emotion words were more pronounced and widespread in LTAA with EXT relative to LTAA without EXT. In Fig. 3,

Table 3. Demographics, Lifetime DSM-IV Diagnoses, and Lifetime Drinking Variables for LTAA With and Without a Lifetime EXT Diagnosis

\begin{tabular}{|c|c|c|c|}
\hline Measures & $\begin{array}{l}\text { LTAA without } \\
\text { EXT }(n=51) \\
\% \text { or } M(\mathrm{SD})\end{array}$ & $\begin{array}{l}\text { LTAA with } \\
\text { EXT }(n=32) \\
\% \text { or } M(\mathrm{SD})\end{array}$ & $\begin{array}{c}\text { LTAA without } \\
\text { EXT vs. LTAA } \\
\text { with EXT } \\
\text { odds or effect }\end{array}$ \\
\hline \multicolumn{4}{|l|}{ Demographics } \\
\hline $\begin{array}{l}\text { Gender } \\
\text { (\% women) }\end{array}$ & $47 \%$ & $41 \%$ & 0.33 (ns) \\
\hline Age (years) & $49.76(6.49)$ & $47.13(6.62)$ & $5.03^{\star}$ \\
\hline Education (years) & $13.53(2.17)$ & $13.34(2.47)$ & $<0.01$ (ns) \\
\hline \multicolumn{4}{|c|}{ Lifetime DSM-IV diagnoses } \\
\hline $\begin{array}{l}\text { Substance use } \\
\text { disorder }\end{array}$ & $37 \%$ & $63 \%$ & $5.07^{*}$ \\
\hline $\begin{array}{l}\text { Internalizing } \\
\text { disorder }\end{array}$ & $63 \%$ & $59 \%$ & 0.09 (ns) \\
\hline \multicolumn{4}{|c|}{ Lifetime drinking variables } \\
\hline $\begin{array}{l}\text { Family history of } \\
\text { alcoholism (\% first- } \\
\text { degree relatives) }\end{array}$ & $37 \%(31 \%)$ & $30 \%(27 \%)$ & 1.35 (ns) \\
\hline $\begin{array}{l}\text { Age of first drink } \\
\text { (years) }\end{array}$ & $14.73(4.64)$ & $13.69(3.33)$ & $1.93(\mathrm{~ns})$ \\
\hline $\begin{array}{l}\text { Lifetime alcohol } \\
\text { use (standard } \\
\text { drinks } \times 1,000)\end{array}$ & $62.13(60.38)$ & $61.50(56.23)$ & 0.05 (ns) \\
\hline $\begin{array}{l}\text { Average alcohol } \\
\text { dose (standard } \\
\text { drinks/month) }\end{array}$ & $197.72(144.29)$ & $224.67(172.83)$ & 2.24 (ns) \\
\hline $\begin{array}{l}\text { Duration of } \\
\text { abstinence } \\
\text { (months) }\end{array}$ & $96.58(90.13)$ & $84.97(76.69)$ & $0.32(\mathrm{~ns})$ \\
\hline
\end{tabular}

EXT, externalizing; LTAA, long-term abstinent alcoholics. $N=83, p$-value not significant at $p<0.05$ (ns), ${ }^{*} p<0.05$. 
LTAA without EXT had lower $\left(d^{\prime}\right)$ values than NSAC in PVB, $F(1,88)=5.76, p \leq 0.05, \eta^{2}=0.06$, but not NVB, $F$ $(1,88)=1.30, \quad p>0.05, \quad \eta^{2}=0.02, \quad$ and MVB, $F(1$, $88)=3.35, p>0.05, \eta^{2}=0.04$. In contrast, LTAA with EXT had lower $\left(d^{\prime}\right)$ values than NSAC in PVB, $F(1,70)=5.52$, $p \leq 0.05, \eta^{2}=0.08, \quad \mathrm{NVB}, \quad F(1,88)=4.38, p \leq 0.05$, $\eta^{2}=0.06$, and MVB, $F(1,88)=7.60, p \leq 0.05, \eta^{2}=0.10$.

\section{DISCUSSION}

Our primary finding was that LTAA had more difficulty discriminating positive, negative, and neutral emotion words than NSAC, and this impairment was in addition to a more general difficulty discriminating words from nonwords. Alcoholics in early abstinence are known to have difficulty judging the valence and intensity of various emotion-laden stimuli, including faces and words (Clark et al., 2007; Foisy et al., 2007a,b; Kornreich et al., 2001; Philippot et al., 1999). Although difficulties decoding emotional facial expressions have been documented in alcoholics with extended abstinence (Fein et al., 2010; Kornreich et al., 2001; Marinkovic et al., 2009), research to date has not established whether LTAA have difficulties processing other types of emotionladen stimuli. Our results showed that specific emotion-word processing impairments were present in LTAA, and these difficulties were larger in individuals with a lifetime EXT disorder diagnosis.

\section{Difficulties with LDM Are Present in LTAA}

Using an LDM task, we showed that accurate and efficient discrimination of words and nonwords were impaired in LTAA. Previous research has shown that acute alcohol intoxication (Marinkovic et al., 2012) and chronic alcoholism (e.g., Glass and Butters, 1985) are associated with less accurate and slower discrimination of words from nonwords and pseudowords. Our findings were consistent with this research, adding that general lexical processing impairments also are present in alcoholics long after they stop using alcohol. To our knowledge, ours is the first study to find that general lexical processing abilities were impaired in alcoholics who (i) do not suffer from Korsakoff's syndrome and (ii) have achieved multiyear abstinence.

\section{Difficulties with Emotion-Word Processing Are Present in LTAA Over and Above Their More General LDM Difficulties}

Using an AGNG task, we showed that accurate discrimination of positive, negative, and neutral emotion words were impaired in LTAA, and these effects could only be partially explained by LTAA's more general lexical processing difficulties. Our findings are consistent with evidence that LTAA have difficulty recognizing the valence of positive, negative, and neutral emotion faces (Fein et al., 2010; Marinkovic et al., 2009) and suggest that alcoholic's emotion processing impairments generalize to emotion words. Our findings also are consistent with evidence that RDA tend to mislabel the valence of positive, negative, and neutral emotional words (Clark et al., 2007) and suggest that this impairment also is present in alcoholics with multiyear abstinence. Moreover, abstinence duration was not associated with emotion-word processing ability in LTAA, suggesting little if any improvement in this functional impairment over time. In the context of previous research, results of the current study could be interpreted to mean that specific emotion-word processing impairments in alcoholics may not fully resolve with extended abstinence.

\section{Lifetime EXT Disorder Diagnoses Are Associated with Greater Difficulty Processing Emotion Words in LTAA}

Results showed that the presence of lifetime internalizing disorder diagnoses were not associated with LTAA's emotion-word processing difficulties. In contrast, lifetime EXT disorder diagnoses were associated with LTAA's emotionword processing difficulties. Specifically, while LTAA without EXT only had lower positive and neutral word discriminability than NSAC, LTAA with EXT had lower positive, negative, and neutral word discriminability than NSAC. Our results are consistent with previous findings that emotional facial expression decoding is impaired in those with attention-deficit/hyperactivity disorder (Pelc et al., 2006), elevated interpersonal problems, and history of risky sexual behaviors (e.g., HIV infection) (Schulte et al., 2011). In the context of previous research, results of the current study suggest that LTAA's difficulty perceiving the emotional content in word stimuli could be associated with a broader propensity for EXT behavior problems, rather than specific to alcoholism per se.

Our results also suggested that LTAA's difficulty with emotion-word processing was associated with their lifetime alcohol burden. Specifically, lower discriminability for positive, negative, and neutral words in LTAA was associated with an earlier age of first drink and higher lifetime alcohol use. Given that alcoholics with co-occurring EXT psychopathology are known to have a more chronic and severe course of alcohol use problems, the current study's findings suggest that emotion-word processing difficulties in LTAA could be associated with both a preexisting vulnerability to EXT disorders and the consequences of heavy use.

\section{Characterization of Emotion-Word Processing Difficulties in LTAA}

Results of the current study provide support for the hypothesis that semantic or affect labeling difficulties play a critical role in alcoholic's impaired perception of emotionally laden words. Maurage and colleagues (2009) proposed that emotion perception is not independent of language, and thus, difficulty rating or classifying emotions could be associated with an underlying linguistic bias or affect labeling impairment. However, Maurage and colleagues (2009) did not find 
support for this affect labeling hypothesis using written scenarios (i.e., emotional scripts). Our findings provide some support for this hypothesis, indicating that LTAA's poor discriminability of positive, negative, and neutral emotion words could be partially explained by their poor discriminability of words and nonwords.

Nonetheless, our findings also indicated that specific emotion perception impairments are present in LTAA above and beyond their more general lexical processing impairments. That is, after removing the variance in emotion-word processing abilities associated with general lexical processing abilities, LTAA were still poorer than NSAC at discriminating positive from neutral- and negative-valenced words. Furthermore, LTAA with a previously diagnosed EXT disorder had poorer discrimination of emotion words than NSAC regardless of emotion-word valence. This pattern of results could be interpreted to mean that while sematic or affect labeling impairments are partial mediators of emotion-word processing impairments in LTAA, residual emotion perception impairments also are present in LTAA, especially those with a history of EXT disorder.

\section{Study Limitations}

There were a number of limitations in the current research. First, the current design was cross-sectional. This limited our ability to determine whether (i) emotion processing difficulties resolve with extended abstinence or (ii) persons with emotion processing difficulties cannot achieve multiyear abstinence. A longitudinal design that followed alcoholics from the point of detoxification through multiyear abstinence would provide valuable information about the magnitude and extent to which emotion-word processing difficulties resolve with extended abstinence. Second, our conclusions are limited by the groups studied. The current study is silent on the nature and extent to which alcoholics in early stages of recovery have difficulty processing emotional words. Without additional samples, we are unable to definitively conclude whether the current evidence of emotionword processing impairments in LTAA is associated with (i) factors that predispose to alcoholism, (ii) the consequences of chronic alcoholism, or (iii) both. Data from short-term abstinent and actively using alcoholics would shed additional light on the etiology and time course of emotion processing impairments in alcoholics with extended abstinence.

Notwithstanding its limitations, the current research made 2 key observations. First, emotion-word processing impairments were found in alcoholics with multiyear abstinence, and these impairments were over and above LTAA's more general lexical processing impairments. This demonstrates that LTAA's difficulties with emotion perception are more general then previously known, including both emotionladen faces and emotion-laden words. Second, emotion-word processing difficulties in LTAA were independent of abstinence duration and most apparent in those with a previous EXT diagnosis. This suggests that individuals that present alcohol use disorders and other co-occurring EXT disorders are prone to emotion processing difficulties that may not fully recover with extended abstinence. We are following up on these results with additional samples, other emotion information processing tasks, and more sophisticated computational modeling approaches.

\section{ACKNOWLEDGMENT}

This research was supported by National Institutes of Health, NIH grant \#AA016944 to George Fein.

\section{REFERENCES}

American Psychiatric Association (2000) DSM-IV-TR: Diagnostic and Statistical Manual of Mental Disorders. American Psychiatric Publishing, Washington, DC.

Bartsch AJ, Homola G, Biller A, Smith SM, Heinz-Gerd W, Wieset AL (2007) Manifestations of early brain recovery associated with abstinence from alcoholism. Brain 130:36-47.

Bauer D, Cox WM (1998) Alcohol-related words are distracting to both alcohol abusers and non-abusers in Stroop color-naming task. Addiction 93:1539-1542.

Bogg T, Finn PR (2010) A self-regulatory model of behavioral disinhibition in late adolescence: integrating personality traits, externalzing psychopathology, and cognitive capacity. J Pers 78:441-470.

Clark US, Oscar-Berman M, Shagrin B, Pencina M (2007) Alcoholism and judgments of affective stimuli. Neuropsychology 21:346-362.

Endres MJ, Rickert ME, Bogg T, Lucas J, Finn PR (2011) Externalizng psychopathology and behavioral disinhibtion: working memory mediates signal discriminability and reinforcement moderates response bias in approach-avoidance learning. J Abnorm Psychol 120:336-351.

Fein G, Key K, Syzmanski MD (2010) ERP and RT delays in long-term abstinent alcoholics in processing of emotional expressions during gender and emotion categorization tasks. Alcohol Clin Exp Res 35:1127-1139.

Fein G, Torres J, Price LJ, Di Sclafani V (2006) Cognitive performance in long-term abstinent alcoholic individuals. Alcohol Clin Exp Res 30:15381544 .

Finn PR (2002) Motivation, working memory, and decision making: a cognitive-motivational theory of personality vulnerability to alcoholism. Behav Cogn Neurosci Rev 1:183-205.

Foisy ML, Kornreich C, Fobe A, D'Hondt L, Pelc I, Hanak C, Verbanck P, Philippot P (2007a) Impaired emotional facial expression recognition in alcohol dependence: do these deficits persist with midterm abstinence? Alcohol Clin Exp Res 31:404 410.

Foisy ML, Kornreich C, Petiau C, Parez A, Hanak C, Verbanck P, Pelc I, Philippot P (2007b) Impaired emotional facial expression recognition in alcoholics: are these deficits specific to emotional cues? Psychiatry Res 150:33-41.

Frigerio E, Burt DM, Montagne B, Murray LK, Perrett DI (2002) Facial affect perception in alcoholics. Psychiatry Res 113:161-171.

Gilman JM, Homer DW (2008) Modulation of brain response to emotional images by alcohol cues in alcohol-dependent patients. Addict Biol 14:423434

Glass AL, Butters N (1985) The effect of associations and expectations on lexical decision making in normals, alcoholics, and alcoholic Korsakoff patients. Brain Cogn 4:465-476.

Gotlib IH, Krasnoperova E, Yue DN, Joormann J (2004) Attentional biases for negative interpersonal stimuli in clinical depression. J Abnorm Psychol 113:121-135.

Greene DM, Swets JA (1966) Signal Detection Theory and Psychophysics. Wiley, New York. Reprinted 1974 by Krieger, Huntington, NY.

Kornreich C, Blairy S, Philippot P, Hess U, Noel X, Streel E, Le Bon O, Dan B, Pelc I, Verbanck P (2001) Deficits in recognition of emotional 
facial expression are still present in alcoholics after mid- to long-term abstinence. J Stud Alcohol 62:533-542.

MacMillan NA, Creelman CD (1990a) Characteristics of detection theory, threshold theory, and "nonparametric" indexes. Psychol Bull 107:401-413.

MacMillan NA, Creelman CD (1990b) Detection Theory: A User's Guide. Cambridge University Press, New York.

Mann K, Gunther A, Stetter F, Ackermann K (1999) Rapid recovery from cognitive deficits in abstinent alcoholics: a controlled test-retest study. Alcohol Alcohol 34:567-574.

Marinkovic K, Oscar-Berman M, Urban T, O'Reilly C, Howard J, Sawyer K, Harris GJ (2009) Alcoholism and dampened temporal limbic activation to emotional faces. Alcohol Clin Exp Res 11:1880-1892.

Marinkovic K, Rosen BQ, Cox B, Kovacevic S (2012) Event-related theta power during lexical-semantic retrieval and decision conflict is modulated by alcohol intoxication: anatomically constrained MEG. Front Psych 3:1-12.

Maurage P, Campanella S, Philippot P, Charest I, Martin S, de Timary P (2009) Impaired emotional facial expression decoding in alcoholism is also present for emotional prosody and body postures. Alcohol Alcohol 44: 476-485.

Maurage P, Campanella S, Philippot P, Martin S, de Timary P (2008) Face processing in chronic alcoholism: a specific deficit for emotional features. Alcohol Clin Exp Res 32:600-606.

Monnot M, Nixon S, Lovallo W, Ross E (2001) Altered emotional perception in alcoholics: deficits in affective prosody comprehension. Alcohol Clin Exp Res 25:362-369.

Murphy FC, Sahakain BJ, Rubinsztien JS, et al. (1999) Emotional bias and inhibitory control processes in mania and depression. Psychol Med 29:1307-1321.

Nixon SJ, Glenn SW (1995) Cognitive, psychosocial performance and recovery in female alcoholics, in Recent Developments in Alcoholism. Vol. 12. Alcoholism and Woman (Galanter M ed), pp 287-308. Plenum Press, New York.

Oscar-Berman M, Hancock M, Mildworf B, Hutner N, Weber DA (1990) Emotional perception and memory in alcoholism and aging. Alcohol Clin Exp Res 14:383-393.
Pelc K, Kornreich C, Foisy ML, Dan B (2006) Recognition of emotional facial expressions in attention-deficit hyperactivity disorder. Pediatr Neurol 35:93-97.

Philippot P, Kornreich C, Blairy S, Baert I, Den Dulk A, Le Bon O, Streel E, Hess U, Pelc I, Verbanck P (1999) Alcoholics' deficits in the decoding of emotional facial expression. Alcohol Clin Exp Res 23:1031-1038.

Robbins TW, James M, Owen AM, Sahakian BJ, McInnes L, Rabbitt P (1994) Cambridge Neuropsychological Test Automated Battery (CANTAB): a factor analytic study of a large sample of normal elderly volunteers. Dementia 5:266-281.

Robins LN, Cottler L, Buckholz K, Compton W (1998) The Diagnostic Interview Schedule for DSM-IV. Washington University School of Medicine, St. Louis, MO.

Rosenbloom MJ, Pfefferbaum A, Sullivan EV (2004) Recovery of short-term memory and psychomotor speed but not postural stability with long-term sobriety in alcoholic woman. Neuropsychology 18:589-597.

Schulte T, Muller-Oehring EM, Sullivan EV, Pfefferbaum A (2011) Disruption of emotion conflict processing in HIV invection with and without alcoholism comorbidity. J Int Neuropsychol Soc 17:537-550.

SPSS Inc. (2004) SPSS 13.0 for Windows, 13.0 ed. SPSS Inc., Chicago, IL.

Steinmetz JP, Federspeil C (2012) Alcohol-related cognitve impairments and affective impairments in a sample of long-term care residents. Gero Psych 25:83-95.

Stormark KM, Laberg JC, Nordby H, Hugdahl K (2000) Alcoholics' selective attention to alcohol stimuli: automated processing? J Stud Alcohol 61:18-23.

Sullivan EV, Pfefferbaum A (2005) Neurocircuitry in alcoholism: a substrate of disruption and repair. Psychopharmacology 180:583-594.

Townshend JM, Duka T (2003) Mixed emotions: alcoholics' impairments in the recognition of specific emotional facial expressions. Neuropsychologia 41:773-782.

Uekermann J, Daum I, Schlebusch P, Trenckmann U (2005) Processing of affective stimuli in alcoholism. Cortex 41:189-194. 